International Journal of Modern Physics A

(c) World Scientific Publishing Company

Vol. 17, No. 6\&7 (2002) 920-925

\title{
CASIMIR ENERGY AND VACUA FOR SUPERCONDUCTING BALL IN SUPERGRAVITY
}

\author{
ALEXANDER BURINSKII \\ Gravity Research Group, NSI, Russian Academy of Sciences, B. Tulskaya 52. \\ Moscow 113191, Russia \\ Received (received date) \\ Revised (revised date)
}

\begin{abstract}
Casimir energy for solid conducting ball is considered on the base of some finite models. One model is physical and built of a battery of parallel metallic plates. Two finite models are based on the Higgs model of superconductivity. One of them is supersymmetric and based on the Witten field model for superconducting strings. Treatment shows that contribution of Casimir energy can be very essential for superdence state in the neutron stars and nuclear matter.
\end{abstract}

\section{Introduction}

Starting from the first paper by Casimir 1 on this subject, the problem of vacuum energy for (super)conducting ball acquires the troubles caused by divergences by use the perturbative approaches. The divergent vacuum volume term $2 \times \frac{1}{2} \sum_{k} \omega_{k}$ is usually ignored in the field theory and when determining the Casimir effect with plates and shells. Meanwhile, for island systems there "appears some doubt" 2 3 concerning the correctness of the removal of the zero-point term by the way of traditional regularization procedures 3 . Casimir was the first who pointed out on the possible effect of the volume vacuum term. In 1 , analyzing the classical Dirac electron model, he considered two versions of the effect: volume effect with conducting solid ball and surface effect with conducting sphere. In the case of sphere the vacuum zero-point field exists outside the sphere as well as inside. In the case of ideal conducting ball the interior vacuum modes must be entirely excluded, resulting in the infinite jump of the vacuum energy density on the surface of the ball

$$
\Delta E_{\text {vac }}=E_{\text {in }}-E_{\text {out }}=0-V^{-1} \sum_{k}^{\infty} \omega_{k}=-\lim _{k_{\max } \rightarrow \infty} k_{\max }^{4} / 8 \pi^{2}
$$

The necessity of the explicit introduction of the cut-off parameter $k_{\max }$ compelled Casimir to abandon this volume effect and prefer the effect with sphere, where the above divergence is cancelled. 
We consider here some related to this problem models where the result turns out to be finite, and we are going to conclusion that contribution of the Casimir energy can be very essential for superdense matter in the neutron stars and nuclear systems.

\section{Some models leading to finite Casimir energy for conducting ball}

\subsection{Interatomic separation as a cut-off parameter}

A natural cut-off parameter is connected with the real properties of material since the real conductors prove to be transparent for high-frequency modes with $k>k_{\max } \sim \pi / d$, where $d$ is of order of interatomic separation. Consequently, one determines the jump (11) of the vacuum field energy, the low-frequency terms must be taken into account only. It leads to finite result $\Delta E_{v a c}=E_{\text {in }}-E_{\text {out }}=$ $V^{-1} \sum_{k}^{k_{\max }} \omega_{k}-V^{-1} \sum_{k}^{\infty} \omega_{k}=-V^{-1} \sum_{k}^{k_{\max }} \omega_{k}$, since matter is practically transparent for high-frequency modes. Note some peculiarities of the volume Casimir effect for solid ball:

- Unlike the surface Casimir effect, it has a little sensitivity to geometry of the boundary surface and its total energy is additively connected with the volume, occupied by material.

- It gives negative contribution and depends upon the fourth power of interatomic separation $d$, while the usual matter is proportional $d^{-3}$. Hence, when compressing the matter, it increases faster than matter density.

There exist a bound when the matter density $E_{m}=m d^{-3}$ ( $\mathrm{m}$ is the mass of particle) and the vacuum contribution $\Delta E_{v a c}$ are equal and can be compensated:

$$
E_{t o t}=E_{m}+\Delta E_{v a c}=m d^{-3}-\pi^{2} d^{-4} / 8 \approx 0 .
$$

This bound corresponds to 'superclosely packed' particles when the separations between them are $d \approx \pi^{2} / 8 m$, of the order of their Compton length. Further compression of the matter could be impossible without destruction of particles according to the principles of QED.

It shows that the volume Casimir effect can be strong for island systems of superdense matter, and, in the absence of the other withstanding effects, the existence of a superdense pseudovacuum state of matter corresponding to $E_{t o t} \approx 0$ could be expected.

\subsection{One calculable and experimentally verified model}

The considered above model is based on the rough estimations. However, it can be modified turning into a finite, calculable and experimentally verified model. The Casimir effect with parallel plates is computable, it does not contain the connected with structure of matter cut-off parameter and is experimentally supported. The 
negative zero-point energy contribution to intermediate space between plates is

$$
\Delta E_{v a c}=-\pi^{2} / 720 a^{4}
$$

where $a$ is interplate separation.

Solid ball can be modelled by a battery of parallel plates. In this case the volume Casimir effect depends on separations between plates $a$ and can be experimentally checked.

On the other hand, in the assumption that the thickness of the plates is much smaller than interplate separations, the total vacuum contribution will be determined by the value (3). In fact, form of the model (ball or maybe box) is not essential since the dependence on the form can be attributed to the corresponding surface Casimir effect.

\subsection{Higgs field model for superconducting ball}

Instead of the step cut-off function for $k>k_{m p t}$, a smooth decreasing of the permitivity upon the frequency can be considered 6 . For high frequencies $\epsilon(\omega)=$ $1-4 \pi N e^{2} / m \omega^{2}$, where $e$ is charge of particles providing conductivity, $m$ their mass, $N$ the density. Maxwell equations yield then for the transverse components of vector potential

$$
\left(\square+M^{2}(r)\right) U=0,
$$

where

$$
M^{2}(r)=4 \pi N e^{2} / m
$$

inside the conducting ball, and $M=0$ in external region. If we set $\phi(r)=M(r) / e$, the equation (44) can be treated as consequence of the Higgs model of superconductivity where electromagnetic field acquires mass $M$, interacting with the Higgs field $\Phi(r) \neq 0$ inside the ball. The low-frequency modes with $\omega<M$ are 'pushed out' of the ball, and the solutions inside the ball fall off exponentially. The effective energy-momentum tensor depends upon coordinate $r$ and is determined by the regularization relative to the region outside the ball $T_{\text {eff }}^{\mu \nu}(r)=<0\left|T^{\mu \nu}(r)\right| 0>$ $-<0\left|T^{\mu \nu}(\infty)\right| 0>$. The substraction has to be carried out 'mode in mode' 1 , when for separate mode we have $T_{\mu \nu}\left(U_{m}, U_{m}^{*}\right)=\partial_{\mu} U_{m} \partial_{\nu} U_{m}^{*}-\frac{1}{2} g_{\mu \nu} \partial_{\rho} U_{m} \partial^{\rho} U_{m}^{*}-$ $\frac{1}{2} M^{2}(r) g_{\mu \nu}\left|U_{m}\right|^{2}$. The orthonormal set of basis functions $U_{m}(x)$ is determined by

the smooth matching of the solutions outside and inside the ball. As a result the regularized value of energy density inside the ball integrated over $\omega$ to $\omega_{\max }$ is

$$
\Delta E_{v a c}\left(\omega_{\max }\right)=-M^{4} / 32 \pi^{2}-M^{2}\left(\omega_{\max }^{2}-M^{2}\right) / 8 \pi^{2} .
$$

It is quadratically divergent. This means that matter influences not only the lowfrequency modes with $\omega<M$ but also the high-frequency modes penetrating into the matter, thus leading to their energy density decrease. However, simultaneously the increasing of material energy takes place in the process of field-particles interaction 8 . For plane waves with momentum $k$ and polarizations $\alpha$ the one-particle 
energy change is $\delta \epsilon=\sum_{k \alpha} \frac{e^{2}}{2 m \omega}\left|A_{k \alpha}^{2}\right|$. The resulting alteration of material energy density will also be divergent

$$
\Delta E_{\text {int }}=\frac{2 \pi e^{2} N}{m(2 \pi)^{3}} \int_{M}^{\omega_{\max }} \frac{d k^{(3)}}{\omega}=M^{2}\left(\omega_{\max }^{2}-M^{2}\right) / 8 \pi^{2}
$$

and compensates the divergent term of (6) leading to the finite result

$$
\Delta E_{v a c}=-M^{4} / 32 \pi^{2}=-\frac{1}{2} N^{2} e^{4} / m^{2} .
$$

Cancelation of divergent terms is consequence of a hidden supersymmetry in this model.

\section{Supersymmetric Superconducting Ball Model}

In previous model the Higgs field $\phi(r)$ was introduced by hand, filling the ball interior. In the similar soliton-like solutions, "lumps" and "Q-balls", the scalar field $\phi(r)$ concentrates inside the island systems that is controlled by a special nonlinear potential $V(\phi)$. The systems of this kind we will call "type A" -systems. In the Higgs model the scalar field $\phi_{A}(r)$ is complex and gives mass $M(r)=e^{-1} \phi_{A}(r)$ to electromagnetic field $A_{\mu} \quad\left(F_{A \mu \nu}\right)$ inside the ball

$$
L_{A}=-\frac{1}{4} F_{A}^{\mu \nu} F_{A \mu \nu}-2\left(D^{\mu} \phi\right) \overline{\left(D_{\mu} \phi\right)}-V \phi,
$$

where $D_{A}^{\mu}=\nabla_{\mu}+i e A_{\mu}$. The known ball-like models do not contain gauge fields, and it is apparently not possible to construct the consistent type A model with long range external electromagnetic field and finite total energy.

On the other hand, the known models based on the Higgs field (like bags, domain walls, bubbles and strings) display usually a "dual" behavior (say "type B") in the sense that the field $\phi_{B}(r)$ forms a bag or stringlike cavity going to a constant vacuum value $\phi_{B}=\phi_{v a c} \neq 0$ in external region. The finite energy demand is usually satisfied, however the gauge field $B_{\mu}$ acquires mass and becomes short range in external region. We call this system as "type B" one. It corresponds to $A \rightarrow B$ substitution in (9). Thus, none of the systems $A$ or $B$ can separately provide necessary demands for description of superconducting ball.

The exit can be found out in the Witten $U(I) \times \tilde{U}(I)$ field model which was used for description the cosmic superconducting strings $\mathrm{B}$. We use the supersymmetric version of the Witten field model 10 which yields PBS-saturated solutions 11 and supersymmetric vacuum states which are free from quantum corrections.

The Witten field model represents a doubling of the Higgs model and contains the both sectors: type $\mathrm{A}$ and $\mathrm{B}$. The long range electromagnetic fields $A_{\mu}$ acquires mass from field $\phi \equiv \phi_{A}$ filling the ball, and the gauge field $B_{\mu}$ is confined inside the bag formed by scalar field $\sigma \equiv \phi_{B}$. Supersymmetric version of the Witten field model has effective Lagrangian $L=-2\left(D_{A}^{\mu} \phi\right) \overline{\left(D_{A \mu} \phi\right)}-2\left(D_{B}^{\mu} \sigma\right)\left(\overline{D_{B \mu} \sigma}\right)-\partial^{\mu} Z \partial_{\mu} \bar{Z}-$ $\frac{1}{4} F_{A}^{\mu \nu} F_{A \mu \nu}-\frac{1}{4} F_{B}^{\mu \nu} F_{B \mu \nu}-V(\sigma, \phi, Z)$, where the potential $V=\sum_{i=1}^{5}\left|\partial_{i} W\right|^{2}$. The 
superpotential $W\left(\Phi^{i}\right)$ is a holomorphic function of the fife complex chiral fields $\Phi^{i}=\{Z, \phi, \bar{\phi}, \sigma, \bar{\sigma}\}$

$$
W=\lambda Z\left(\sigma \bar{\sigma}-\eta^{2}\right)+(c Z+m) \phi \bar{\phi} .
$$

In the effective Lagrangian the "bar" is identified with complex conjugation, so there are really only three independent scalar fields and the "new" ( neutral ) fields $\mathrm{Z}$ provides the synchronization of the phase transition. The supersymmetric vacuum states corresponding to the lowest value of the potential are determined by the conditions $\partial_{i} W=0$. It yields two supersymmetric vacuum states with $V=0$ :

$$
\text { I) } \quad Z=0 ; \quad \phi=0 ; \quad|\sigma|=\eta ; \quad W=0
$$

we set it for external vacuum; and

$$
\text { II) } \quad Z=-m / c ; \quad \sigma=0 ; \quad|\phi|=\eta \sqrt{\lambda / c} ; \quad W=\lambda m \eta^{2} / c=m \phi^{2},
$$

we set it as a state inside the bag.

The treatment of the gauge field $A_{\mu}$ and $B_{\mu}$ in $B$ is similar in many respects because of the symmetry between $A$ and $B$ sectors allowing one to consider the state I ) in outer region as superconducting one in respect to the gauge field $B_{\mu}$, which provides confinement of the $B_{\mu}$ field inside the bag. ${ }^{a}$

One can check the phase transition in the planar wall approximation neglecting the gauge fields. Using the Bogomol'nyi transformation one can represent the energy density as follows

$$
\rho=\frac{1}{2} \delta_{i j}\left[\Phi^{i},{ }_{z}+\frac{\partial W}{\partial \Phi^{j}}\right]\left[\Phi^{j},{ }_{z}+\frac{\partial W}{\partial \Phi^{i}}\right]-\frac{\partial W}{\partial \Phi^{i}} \Phi^{i},{ }_{z},
$$

where the last term is full derivative. Then, integrating over the wall depth $z$ one obtains for the surface energy density of the wall $\epsilon=\int_{0}^{\infty} \rho d z=\frac{1}{2} \int \Sigma_{i}\left(\Phi^{i},{ }_{z}+\frac{\partial W}{\partial \Phi^{i}}\right)^{2} d z+$ $W(0)-W(\infty)$. The minimum of energy is achieved when the first-order Bogomol'nyi equations $\Phi^{i},{ }_{z}+\frac{\partial W}{\partial \Phi^{i}}=0$ are satisfied. Its value is given by $\epsilon=W(0)-W(\infty)=$ $m \phi_{A(i n)}^{2}$. Therefore, this domain wall is BPS-saturated solution, and corresponding vacuum states are supersymmetric and do not acquire quantum corrections. Supersymmetric vacuum state inside the ball has energy density $\rho=V / 2=0$, in spite of the nonzero density of scalar field there, displaying analogue with (2). In supergravity this problem is connected with the regular black hole and particlelike models 11.13 when singularity is replaced by a superconducting source. The extra contribution to potential in $N=1$ supergravity can yield negative vacuum energy 12

$$
\rho=-\frac{3}{2} W \bar{W}=-\frac{3}{2} m^{2} \phi^{4}
$$

(here $m$ is parameter of supersymmetric model) leading to AdS vacuum state inside the source.

aThere are some evidences that in B-sector a "dual" type of superconductivity has to be realized. 


\section{Conclusion}

The negative contribution of the Casimir zero-point energy for (super)conducting ball turns out to be finite and calculable in represented models, showing its importance for island systems with superdense matter.

\section{Acknowledgments}

Author would like to thank Organizing Committee of the Conference, and in particular Prof. Michael Bordag, for very kind invitation and financial support.

\section{References}

1. H.B.G. Casimir,Physica XIX(1956) 846.

2. C. Itzikson and I.C.Zuber, Quantum Field Theory(McGraw Hill, New York, 1980).

3. N.D. Birell and P.C.W. Davies, Quantum Fields in Curved Space (Cambridge U.P.,1982).

4. B.S. De Witt, Phys. Rep.19(1975) 295.

5. K.A.Milton, Ann.Phys. (NY)(1980) 49.

6. I.Schwinger, L. De Raad and K.A. Milton, Ann.Phys.115(1978) 1.

7. A. Burinskii, Phys.Lett. B216(1989) 123.

8. V.B. Berestetskii, E.M. Lifshits and A. Pitayevskii, Relativistic Quantum Theory. Part 1 (Moscow, Nauka, 1968).

9. E. Witten, Nucl.Phys. B249(1985)557.

10. J.R. Morris, Phys.Rev.D 53(1996)2075.

11. A. Burinskii, in:Noncommutative Structures in Mathematics and Physics, eds. S.Duplij and J.Wess ,(Kluwer Academic Publishers, 2001) p.181, hep-th/0011188.

12. M. Cvetič and H. Soleng, Phys. Rept.282(1997)159.

13. A. Burinskii, Class. Quantum Grav.16(1999)3497, hep-th/0008129. 